\section{Associação Entre Carboidratos da Dieta Habitual e Diabetes Mellitus Tipo 2: Evidências Epidemiológicas}

\section{RESUMO}

A prevalência do diabetes tem se elevado vertiginosamente e a dieta habitual é um dos principais fatores determinantes passiveis de modificação na prevenção de doenças crônicas não-transmissíveis (DCNT). Evidências sobre o papel da qualidade dos carboidratos da dieta no risco para o diabetes tipo 2 têm sido consideradas inconsistentes. O presente estudo de revisão da literatura analisa evidências epidemiológicas da associação entre a qualidade dos carboidratos da dieta habitual e risco de diabetes em adultos. Embora alguns estudos indiquem um efeito de risco de dietas com elevados teores de índice glicêmico e pobre em fibras para o diabetes, os resultados são controversos e há indícios de um efeito mediado pelo magnésio contido na casca dos grãos, enfatizando-se a relevância da analise do consumo de alimentos em detrimento de nutrientes isoladamente em investigações sobre dieta e risco para DCNT. As evidências sugerem que uma dieta rica em cereais integrais e vegetais, em detrimento do consumo de cereais refinados, sacarose e frutose, possa exercer um papel protetor para o diabetes. Entretanto, um maior número de ensaios clínicos aleatorizados são necessários para o estabelecimento das hipóteses causais e plausabilidade biológica. (Arq Bras Endocrinol Metab 2006;50/3:415-426)

Descritores: Consumo alimentar; Carboidratos; Índice glicêmico; Fibra dietética; Epidemiologia do diabetes

\begin{abstract}
Association Between Dietary Carbohydrates and Type 2 Diabetes Mellitus: Epidemiological Evidences.

The prevalence of type 2 diabetes has been escalating throughout the world and usual food consumption is regarded as one of the most important environmental determinants for chronic diseases. Thus, the role of type and quality of carbohydrates on the etiology of type 2 diabetes is still poorly understood. The present review discusses the available epidemiological evidence regarding the effect of the type and source of carbohydrate of the usual diet and the risk of type 2 diabetes in adults. Although epidemiological investigations support the hypothesis for a potential risk effect of high glycemic index and low fiber content diets for diabetes, the results are controversial and the benefit may be related to the magnesium content of the structure of the grains, suggesting the relevance of taking into account the food sources instead of nutrients on investigations of diet and risk of chronic diseases. The available evidence suggests that eating a diet rich in whole grain cereals and vegetables and low in refined grains, sucrose and fructose contents is beneficial in the prevention of diabetes. Thus, more clinical trials are required to support the associations and establish biologically plausible pathways. (Arq Bras Endocrinol Metab 2006;50/3:415-426)
\end{abstract}

Keywords: Food consumption; Carbohydrates; Glycemic index; Dietary fiber; Diabetes epidemiology

\section{revisão}

Daniela S. Sartorelli Marly A. Cardoso

Departamento de Medicina Social da Faculdade de Medicina de Ribeirão Preto (DSS) e Departamento de Nutrição da Faculdade de Saúde Pública (MAC), Universidade de São Paulo, SP. 
$\mathbf{E}^{\infty}$ M MUITOS PAÍSES DO MUNDO a prevalência do diabetes mellitus tipo 2 (DM) tem se elevado vertiginosamente e espera-se um incremento ainda maior. Nos países em desenvolvimento há uma tendência de aumento na freqüência em todas as faixas etárias, especialmente nas mais jovens, cujo impacto negativo sobre a qualidade de vida e a carga da doença aos sistemas de saúde é imensurável (1).

De acordo com relatório recente da Organização Mundial da Saúde (OMS) sobre dieta, nutrição e prevenção de doenças crônicas não-transmissíveis (DCNT), o consumo alimentar habitual constitui um dos principais fatores determinantes passíveis de modificação para DCNT (2). Embora as evidências epidemiológicas demonstrem um potencial efeito protetor do elevado consumo de fibras e teores reduzidos de índice glicêmico da dieta habitual para o diabetes (2), a influência da qualidade dos carboidratos na etiologia dos distúrbios do metabolismo da glicose ainda é pouco compreendida (3). Conforme a Associação Americana de Diabetes (ADA), as evidências de uma relação causal do índice glicêmico e carga glicêmica da dieta em relação à prevenção do diabetes são inconsistentes, sendo necessário maior número de investigações científicas, em especial ensaios clínicos aleatorizados (4).

A presente revisão da literatura discute as evidências epidemiológicas da associação entre a qualidade dos carboidratos da dieta, diabetes e doenças associadas.

Para a seleção dos artigos adotou-se as bases de dados do Medlars Online (MEDLINE) e Literatura Latino-Americana e do Caribe de Informação em Ciências da Saúde (LILACS) com as palavras-chave em inglês no resumo: "diet", "food consumption", "carbohydrates", "fiber", "glycemic index", "glycemic load", "fructose", "sucrose", "lactose", "grains", "cereals" associadas aos termos "prevention" e "diabetes". Foram incluídos os estudos epidemiológicos (retrospectivos ou prospectivos) sobre a quantidade e/ou qualidade dos carboidratos e risco de diabetes tipo 2 conduzido em adultos publicados no período entre janeiro de 2004 a maio de 2005 . Dada a escassez de estudos epidemiológicos abordando esta temática, estudos transversais que investigaram a associação do consumo com a ocorrência da doença também foram considerados no presente artigo de revisão.

\section{Resposta glicêmica pós-prandial: o papel da qualidade dos carboidratos da dieta}

O conceito do índice glicêmico foi proposto desde 1981 por Jenkins e cols. com o intuito de se caracteri- zar o perfil de absorção dos carboidratos e reposta metabólica após as refeições (5). Consiste em uma escala de resposta glicêmica a uma quantidade fixa de carboidrato $(50 \mathrm{~g})$ quando comparado à resposta glicêmica de um alimento padrão, geralmente glicose ou pães. $\mathrm{O}$ conceito do índice glicêmico pode ser considerado uma extensão da hipótese da fibra dietética, sugerindo que a absorção lenta dos nutrientes de alguns alimentos seria benéfica à saúde. $\mathrm{O}$ índice glicêmico da dieta habitual é um indicador da qualidade do carboidrato da dieta consumida (6).

A carga glicêmica do alimento é o produto do índice glicêmico pela quantidade de carboidratos. A carga glicêmica da dieta seria o resultado do efeito glicêmico da dieta como um todo, sendo uma medida de avaliação da quantidade e qualidade de carboidratos, considerando o efeito na glicemia do consumo de uma porção usual de um alimento (7).

A velocidade de absorção dos carboidratos é diretamente influenciada por outros componentes da dieta, como o teor de lipídeos, proteínas e fibras. O teor de lipídeos dos alimentos retarda o esvaziamento gástrico e a velocidade de liberação dos nutrientes para a corrente sangüínea, reduzindo o pico hiperglicêmico pós-prandial imediato (8). Por outro lado, uma dieta rica em proteínas possui ação direta na hipersecreção de insulina, atenuando a elevação da glicemia após as refeições. Entretanto, sugere-se que este efeito seja dependente da fonte protéica consumida. Em animais, uma maior secreção de insulina foi observada após consumo de proteínas provenientes de laticínios como queijos magros, que são rapidamente digeridos. Por outro lado, este resultado não foi observado quando a fonte protéica avaliada foi albumina de ovos crus, que possui digestibilidade mais lenta (9).

Além da composição química, o tamanho das partículas e o processamento dos alimentos poderão influenciar a velocidade de digestão e absorção dos carboidratos. O menor tamanho das partículas determinado tanto pelo processamento/preparo do alimento como pelo maior tempo de mastigação facilita a digestão e absorção dos carboidratos, interferindo na exposição das moléculas à ação da amilase salivar responsável pela quebra parcial da parede celular do alimento. Além disso, a forma de cocção também poderá interferir na disposição das moléculas e velocidade de absorção. $\mathrm{O}$ amido em presença de água, mediante aquecimento, sofre o processo de gelatinização, facilitando a ação da amilase. Por outro lado, durante o resfriamento do alimento ocorre a retrogradação, que consiste na recristalização das moléculas, impedindo a ação da amilase (3). Um estudo 
experimental conduzido no Brasil demonstrou que a integridade da parede celular dos feijões interfere no aproveitamento do amido, podendo atuar como uma barreira física à gelatinização dos grânulos e à ação das enzimas hidrolíticas. Assim, um maior tempo de cocção das leguminosas poderá facilitar a hidrólise e velocidade de absorção dos carboidratos, aumentando seu índice glicêmico (10). Além do feijão, o rompimento da estrutura da casca de outros alimentos, como a pipoca e a aveia, vem sendo apontado como um fator modificador de seu índice glicêmico, sugerindo a necessidade de se considerar a integridade dos grãos habitualmente consumidos nos estudos epidemiológicos (3).

O efeito das fibras solúveis na redução da velocidade de absorção da glicose vem sendo atribuído tanto ao retardo do esvaziamento gástrico como em decorrência da adsorção e interação com os nutrientes, conferindo uma menor superfície de contato direto com a parede do intestino delgado. A maior resistência à difusão através da mucosa ocorre em virtude da viscosidade conferida ao bolo alimentar de uma dieta rica em fibras (11). Em relação às fibras insolúveis, os dados disponíveis ainda são inconsistentes.

\section{Relação entre a qualidade dos carboidratos e risco para diabetes e doenças associadas: evidências epidemiológicas}

A qualidade dos carboidratos e sua associação com o excesso de peso e resistência à insulina em pesquisa experimental há muito vem sendo sugerida (12). Estudos epidemiológicos recentes sugerem que tanto a quantidade como a qualidade dos carboidratos constituiria um importante fator preditor de dislipidemia, doenças cardiovasculares e diabetes, principalmente entre indivíduos susceptíveis à resistência à insulina, com elevado índice de massa corporal (IMC) $(13,14)$.

\section{Índice glicêmico e carga glicêmica}

Sabe-se que dietas com baixo índice glicêmico promovem sensação de saciedade, prolongando o período de reincidência da fome e reduzindo o consumo calórico nas refeições subseqüentes (15). Este efeito estaria relacionado à hiperinsulinemia e hipoglicemia reacional decorrente da hiperglicemia pós-prandial imediata após o consumo de uma refeição rica em carboidratos de rápida absorção. A hiperinsulinemia promove maior captação tecidual de nutrientes e acúmulo no tecido adiposo em detrimento de sua oxidação, impulsionando ganho de peso corporal (15). Além disso, prorrogando-se o tempo de absorção dos nutrientes pode-se produzir estímulo contínuo aos recep- tores do trato gastrointestinal mediado pela ação de hormônios como a colecistoquinina e peptídeo l semelhante ao glucagon (glucagon-like peptide-1, em inglês) a atuarem nos centros hipotalâmicos de controle da saciedade decorrente da dieta com baixo índice glicêmico. Por outro lado, a hipoglicemia reacional observada após uma refeição com elevado índice glicêmico parece exacerbar a sensação de fome em período reduzido de tempo (16). Desta forma, dietas com elevado índice glicêmico têm sido sugeridas como possível fator desencadeante da obesidade, considerada fator de risco convincente no desenvolvimento de diversas doenças crônicas (2).

Em relação ao risco para doenças cardiovasculares, estudos sugerem uma possível associação positiva com uma dieta habitual de elevado índice glicêmico e carga glicêmica (17). A correlação entre a qualidade dos carboidratos da dieta e importantes fatores de risco de doenças cardiovasculares, como dislipidemia (18), insulina imunorreativa (19), proteína C-reativa (20), síndrome metabólica (21) e obesidade (15) vem sendo assinalada por estudos epidemiológicos recentes.

Sugere-se que uma dieta habitual hiperglicídica seja um possível fator de risco para a dislipidemia, assim como ocorre em dietas hiperlipídicas. Os mecanismos fisiopatológicos ainda não estão bem esclarecidos, mas especula-se que este efeito seria atribuído ao maior estímulo à lipogênese hepática, especialmente na síntese de triglicérides, assim como uma menor depuração do VLDL-colesterol (VLDL-c) através de uma maior oferta de glicose plasmática. Mulheres em período pós-menopausa, indivíduos com obesidade abdominal, hipertensão, hiperinsulinemia e hiperglicemia parecem ser mais susceptíveis à indução de síntese de triglicérides mediado por dieta hiperglicídica (22). Por outro lado, a hipótese de explicação da relação do índice glicêmico da dieta e a proteína Creativa estaria relacionada ao desencadeamento do processo inflamatório (20). Uma outra explicação seria a excreção urinária excessiva de cromo, mineral envolvido no metabolismo de carboidratos e lipídeos, promovida pela hiperglicemia pós-prandial (17).

Um estudo prospectivo conduzido nos Estados Unidos avaliou o efeito da quantidade e qualidade dos carboidratos da dieta habitual na ocorrência de doenças cardiovasculares (DCV) entre 75.000 mulheres de 38-63 anos durante 10 anos de seguimento. O consumo mediano de carga glicêmica ajustado pelas calorias totais foi de 161 unidades diárias. Após ajuste por tabagismo, idade, consumo calórico total da dieta (VCT) e outros fatores de risco, o risco relativo (RR) e intervalo com 95\% de confiança (IC 95\%) para doenças 
cardiovasculares no último quintil de carga glicêmica foi de $1,98(1,41 ; 2,77)$. Em análises estratificadas por IMC, observou-se que o RR (IC95\%) foi maior entre mulheres com IMC > $29 \mathrm{~kg} / \mathrm{m}^{2}[1,74(1,42 ; 1,97)]$ quando comparadas às mulheres com IMC entre $23 \mathrm{e}$ $29 \mathrm{~kg} / \mathrm{m}^{2}[1,2(1,05 ; 2,03)]$ e com IMC $<23 \mathrm{~kg} / \mathrm{m}^{2}$ $[(0,94(1,00 ; 1,11)]$, sugerindo que o papel da qualidade dos carboidratos no risco de doenças cardiovasculares é influenciado pelo peso corpóreo e sensibilidade à insulina da população estudada (13).

Uma importante medida de prevenção do diabetes e controle metabólico consiste no controle glicêmico. A glicemia de jejum é diretamente influenciada pela produção hepática. Por outro lado, a glicemia pós-prandial é modulada principalmente pela velocidade de liberação de carboidratos para a corrente sangüínea após as refeições, pelo tempo de depuração através da síntese de insulina e pela sensibilidade tecidual periférica à sua ação. Desta forma, a quantidade e qualidade dos carboidratos há muito vem sendo considerada um importante fator dietético envolvido neste mecanismo. Em recente meta-análise de ensaios clínicos aleatorizados sobre o efeito do índice glicêmico no controle metabólico de portadores de diabetes, BrandMiller e cols. verificaram que a redução da hemoglobina glicada entre indivíduos com consumo de dietas de baixo IG (média de 65 unidades/dia) foi 7,5\% maior do que o observado em indivíduos com consumo de dietas de elevado IG (média de 83 unidades/dia) (23).

Um estudo epidemiológico prospectivo, Nurses' Health Study, conduzido em 65.173 mulheres americanas com idade entre 40 e 65 anos, demonstrou uma associação positiva entre o consumo de uma dieta habitual com elevado índice glicêmico e a incidência de diabetes após 6 anos de seguimento. A mediana de consumo de índice glicêmico e carga glicêmica foi de 75 e 166 unidades/dia, respectivamente. O RR (IC 95\%) do último quintil de consumo de índice glicêmico foi de $1,37(1,09 ; 1,71)$ quando comparado ao menor quintil de consumo ajustado por possíveis fatores de confusão. Em relação à carga glicêmica, o risco para diabetes no maior quintil de consumo $[R R$ (IC95\%) $1,47(1,16 ; 1,86)]$ apenas foi observado após a inclusão de fibras de cereais no modelo múltiplo, cujo risco relativo do maior consumo foi de 0,72 $(0,58 ; 0,90)$ quando comparado ao menor quintil. Os autores verificaram que as mulheres que consumiram dietas com maior carga glicêmica (último tercil, mediana $\geq 165$ unidades/dia) associada ao menor consumo de fibras de cereais (primeiro tercil, $\leq 2,5 \mathrm{~g} / \mathrm{dia}$ ) apresentaram maior risco de desenvolverem o diabetes [RR (IC 95\%) $2,5(1,14 ; 5,51)]$ quando comparadas às mulheres que referiram dietas habituais com menor carga glicêmica e maiores teores de fibras de cereais, ricas em magnésio (24).

Estudos epidemiológicos sobre a associação entre o consumo alimentar e risco de glicemia de jejum alterada ou tolerância à glicose diminuída são escassos na literatura. Um ensaio clínico aleatorizado cruzado conduzido com 34 indivíduos portadores de tolerância à glicose diminuída (idade entre 30 e 65 anos) avaliou o impacto na glicemia e insulinemia de jejum de quatro dietas distintas: 1 ) dieta hiperglicídica ( $\geq 55 \%$ das calorias provenientes de carboidratos e 30\% de lipídios) com elevado índice glicêmico, 2) dieta hiperglicídica com baixo índice glicêmico, 3) dieta hipoglicídica (consumo de carboidratos $\leq 45 \%$ do calor calórico total), 4) dieta rica em ácidos graxos monoinsaturados $(45 \%$ das calorias de carboidratos, $40 \%$ de lipídios sendo $20 \%$ de monoinsaturados). Os participantes alocados no grupo de dietas de elevado IG foram orientados a consumir uma porção de alimento de elevado IG em cada refeição (pão branco, biscoitos, arroz polido ou batata). Os indivíduos alocados no grupo de dietas de baixo IG foram orientados a consumir uma porção de cereais integrais em todas as refeições diárias (cereais integrais, massas, arroz parboilizado, leguminosas ou sopas instantâneas). Após 4 meses de seguimento, a redução da glicemia 2 horas após sobrecarga de glicose entre os indivíduos que consumiram dieta hipoglicídica foi semelhante aos indivíduos com consumo de dietas de baixo IG, sugerindo importante efeito da quantidade dos carboidratos em detrimento de sua qualidade no controle glicêmico de indivíduos portadores de tolerância à glicose diminuída. Por outro lado, observou-se que a concentração plasmática de insulina de jejum entre indivíduos com consumo de dietas de baixo IG foi $20 \%$ maior do que o verificado entre indivíduos com dietas de elevado IG (25).

Alguns autores sugerem que o efeito benéfico do consumo de uma dieta com baixo índice glicêmico consistiria na redução do pico pós-prandial de insulina e glicose (6), promovendo menor sobrecarga pancreática a longo prazo, cuja falência poderia ser originada por um efeito tóxico direto causado pela hiperglicemia (14). Além disso, sugere-se que os efeitos adversos de uma dieta com elevado índice glicêmico estariam relacionados ao incremento na concentração pós-prandial tardia de ácidos graxos livres, produzindo maior resistência à insulina. Este mecanismo seria desencadeado pela hipersecreção de hormônios contraregulatórios, tais como o cortisol, glucagon e hormônio do crescimento, em decorrência da hipogli- 
cemia reacional promovida por uma dieta de elevado índice glicêmico (26). Neste processo, tanto a quantidade como a qualidade dos carboidratos contido nos alimentos influenciaria a resposta metabólica após o consumo habitual deste alimento (6). No Brasil, um estudo transversal conduzido entre 1.054 nipobrasileiros ( $\geq 30$ anos) de primeira e segunda geração, não miscigenados, verificou uma associação positiva entre IG da dieta habitual e glicemia de jejum entre mulheres portadoras de excesso de peso (IMC $\geq 23$ $\left.\mathrm{kg} / \mathrm{m}^{2}\right)[\beta 1(\mathrm{IC} 95 \%)=0,0000629(0,000002-$ $0,000124), p<0,05]$, independente da idade, geração, escolaridade, prática de atividades físicas, presença de dislipidemia, hipertensão, circunferência de cintura, tabagismo, consumo de álcool, fibras, colesterol e calorias da dieta habitual (28). Entretanto, nenhuma relação entre IG e glicemia de jejum foi verificada entre mulheres eutróficas, concordando com estudos epidemiológicos prévios (13). Entretanto, resultados provenientes de investigações epidemiológicas da relação entre a qualidade dos carboidratos e resistência à insulina são inconsistentes. Embora uma relação positiva entre o índice glicêmico da dieta e o indicador de resistência à insulina, HOMA R, tenha sido observada em análise transversal do estudo de Framingham (21), nenhuma associação entre o índice glicêmico da dieta e HOMA R foi verificada em análises recentes, como no Inter 99 Study, conduzido entre 5.675 adultos dinamarqueses (27).

\section{Fibras dietéticas, cereais integrais e cereais refinados}

Em relação às fibras insolúveis, a suposta ação protetora ainda não é bem elucidada. Conforme mencionado previamente, há evidências de que o consumo habitual de dietas de elevada carga glicêmica e baixos teores de fibras de cereais seja um fator de risco para diabetes, como foi observado no Nurses' Health Study (14). Em análise transversal com dados provenientes do estudo de Framingham, uma relação inversa entre o consumo de cereais integrais e insulina de jejum, razão cinturaquadril e LDL-colesterol (LDL-c) foi observada (29). Entretanto, os modelos de regressão não foram devidamente controlados pelo consumo de magnésio da dieta, considerado importante fator protetor para $\mathrm{o}$ diabetes (3).

Uma análise recente dos dados do Health Professionals Follow-up Study avaliou o papel isolado de componentes dos grãos integrais (casca, endosperma e germe) no risco de doenças cardiovasculares (DCV). Após 14 anos de seguimento, observou-se que o consumo da casca dos grãos representou uma redução de
$28 \%$ no risco de DCV, independente das fibras totais da dieta, sugerindo que o efeito seria mediado pelos nutrientes contidos na casca dos grãos, como os antioxidantes, fitoquímicos e magnésio. Nenhuma associação entre o consumo de endosperma (principal componente dos cereais refinados) e o risco de DCV foi verificada (30).

A relação entre o consumo de cereais integrais e refinados e o risco para diabetes também foi avaliada no Health Professionals Follow-Up Study (31). O consumo mediano de cereais integrais relatado pelos participantes foi de 1,1 porções/dia e cereais refinados de 1,7 porções diárias. Após 12 anos de seguimento, observou-se um RR (IC 95\%) de 0,58 (0,47-0,70) para o último quintil de consumo de cereais integrais quando comparado ao menor quintil de consumo. Entretanto, esta relação não se manteve significante após o ajuste por magnésio, fibras de cereais e IG da dieta, sugerindo que efeito protetor possa estar relacionado a outros componentes de dietas ricas em cereais integrais. Neste estudo, nenhuma relação entre o consumo de cereais refinados e diabetes foi observada.

Um outro estudo prospectivo americano, Iowa Women's Health Study, avaliou a relação entre o consumo de carboidratos, fibra total, magnésio, alimentos fonte de carboidratos, índice glicêmico e incidência de diabetes em 35.988 mulheres após 6 anos de seguimento. Os valores medianos de consumo de índice glicêmico e carga glicêmica da dieta habitual foram de 69 e 120 unidades/dia, respectivamente. Não houve associação entre o consumo de carboidratos totais, grãos refinados, frutas, vegetais, fibra solúvel e índice glicêmico e incidência de diabetes. O RR (IC 95\%) do último quintil de consumo de cereais integrais foi de $0,79(0,65 ; 0,96)$ e de fibra total de $0,78(0,64 ; 0,96)$ quando comparado ao menor quintil de consumo. Em relação ao magnésio, o RR (IC 95\%) de incidência de diabetes no último quintil de consumo foi de 0,67 $(0,55 ; 0,82)$ quando comparado ao menor consumo após ajuste por possíveis fatores de confusão não dietéticos (32).

A associação entre o consumo de fibra dietética, índice glicêmico da dieta habitual e incidência do diabetes também foi investigada em 12.251 adultos afroamericanos e caucasianos. O Atherosclerosis Risk in Communities (ARIC) Study, estudo prospectivo com 9 anos de seguimento, não encontrou associação entre $o$ consumo de fibra total, fibra de frutas, fibra de leguminosas, índice glicêmico ou carga glicêmica e incidência de diabetes, após ajuste por idade, IMC, sexo, centro de tratamento do estudo, escolaridade, tabagismo, atividade física e consumo de fibra de cereais. A razão 
de ocorrência do último quintil de consumo de fibra de cereais foi de $0,75(0,60-0,92)$ em caucasianos quando comparados ao primeiro quintil de consumo, mas não significante em afro-americanos $[0,86(0,65-1,15)]$, sugerindo interação entre fatores genéticos, consumo alimentar e risco para diabetes (33).

$\mathrm{O}$ efeito do consumo habitual de cereais integrais, fibras da dieta e incidência do diabetes também foi avaliado em estudo prospectivo conduzido em 2.286 homens e 2.030 mulheres de 40-69 anos na Finlândia. Após 10 anos de seguimento, observou-se um RR (IC 95\%) do consumo de fibra de cereais de $0,39(0,20-0,77)$ em relação ao menor quintil de consumo. Entretanto, não houve associação entre consumo do grupo de cereais integrais e risco para diabetes [RR 0,65 $(0,36-1,18)]$, após ajuste por fatores de confusão não dietéticos, calorias totais, consumo de vegetais, frutas em geral e frutas vermelhas da dieta habitual (34). Os autores não avaliaram o efeito do magnésio da dieta no risco de diabetes.

No Health Professionals Follow-Up Study, estudo americano prospectivo conduzido em 42.759 homens entre 40 e 75 anos de idade, observou-se associação positiva entre o elevado índice glicêmico da dieta e incidência do diabetes após 6 anos de seguimento. Os valores medianos de consumo de índice glicêmico e carga glicêmica da dieta habitual foram de 73 e 160 unidades/dia. O RR (IC 95\%) do último quintil de consumo de índice glicêmico foi de 1,37 $(1,02 ; 1,83)$ quando comparado ao menor consumo. Entretanto, esta associação tornou-se significante somente após o ajuste por fibra dos cereais. Em relação ao consumo de fibras de cereais, o RR (IC 95\%) do último quintil de consumo foi de $0,7(0,51 ; 0,96)$ quando comparado ao menor consumo, mantendo-se significante mesmo após ajuste por magnésio da dieta. Observou-se que uma dieta habitual com elevado índice glicêmico (último tercil de consumo, mediana $\geq$ 188 unidades/dia) e reduzidos teores de fibras provenientes de cereais (primeiro tercil de consumo, mediana $\leq 3,2 \mathrm{~g} / \mathrm{dia}$ ) representou maior risco de desenvolver diabetes [RR (IC 95\%) 2,17 (1,04; 4,54)]. Nenhuma relação entre o consumo de carboidratos totais e carga glicêmica da dieta e risco de diabetes foi observada (35).

Entre as 91.249 mulheres jovens (20-44 anos) acompanhadas pelo Nurses' Health Study II, elevados valores de índice glicêmico foram associados ao risco para diabetes após 8 anos de seguimento [RR 1,59 $(1,21-2,10)]$. Em relação ao consumo de fibra de cereais, o RR (IC 95\%) foi de 0,64 $(0,48-0,86)$ no último quintil quando comparado ao menor consumo deste nutriente. Nenhuma associação foi observada entre a carga glicêmica da dieta e risco de diabetes nesta população (36). Neste recente estudo epidemiológico, controverso em relação a estudos prévios, o risco para diabetes relacionado ao maior consumo de IG e o efeito protetor do maior consumo de fibras de cereais foram independentes do magnésio da dieta. Nestes modelos de regressão logística, as associações observadas foram independentes da idade, IMC, história familiar de diabetes, atividade física, tabagismo, história de hipertensão arterial (HA) ou dislipidemia, reposição hormonal, consumo habitual de álcool, calorias totais, magnésio, cafeína, fibra de cereais, gorduras totais e frações.

Uma análise transversal do estudo de Framingham (29) avaliou a associação entre o consumo de fibras, cereais e índice glicêmico e resistência à insulina (HOMA R). Efeito protetor foi observado para o consumo do último quintil de consumo de cereais integrais [RR 0,67 $(0,48-0,91)$ ] e fibra de cereais [RR $0,62(0,45-0,86)]$ em relação à resistência à insulina. Por outro lado, o consumo do último quintil de índice glicêmico da dieta foi associado ao maior risco de resistência à insulina $[R R ~ 1,41(1,04-1,91)]$ quando comparado ao menor quintil de consumo. Entretanto, esta associação não se manteve significante após o ajuste por magnésio e fibra total da dieta. Nenhuma associação entre o consumo de carboidratos, fibras totais, fibras de frutas, vegetais ou leguminosas, carga glicêmica ou cereais refinados e prevalência de síndrome metabólica foi verificada (29).

Um estudo transversal, Insulin Resistance Atherosclerosis Study (IRAS), investigou a relação entre o consumo de cereais integrais e sensibilidade à insulina entre indivíduos normoglicêmicos ou portadores de tolerância à glicose diminuída em modelos de regressão linear. Os dados sugerem uma relação inversa entre o consumo deste grupo de alimentos e a insulina após ajuste para fatores de confusão não dietéticos e calorias totais (37).

No Estudo de Diabetes e Doenças Associadas em Nipo-Brasileiros de Bauru, nenhuma relação entre o índice glicêmico, carga glicêmica da dieta e distúrbios do metabolismo da glicose (glicemia de jejum alterada, tolerância à glicose diminuída ou diabetes tipo 2) foi verificada. Por outro lado, o tercil superior de consumo agrupado de fibras provenientes de cereais refinados (arroz polido e pão branco) e frutas/sucos representou um incremento de $70 \%$ na razão das chances (RC) para glicemia de jejum alterada [RC (IC 95\%): 1,70 (1,06-2,74), p tendência $=0,02]$, independente da idade, geração, tabagismo, prática de 
atividades físicas, presença de obesidade abdominal, hipertensão, dislipidemia, consumo de colesterol e calorias da dieta habitual. Entretanto, esta associação não se manteve significante após o ajuste pelo consumo de cereais refinados e frutas. $\mathrm{O}$ último tercil de consumo agrupado de cereais refinados e frutas/sucos estava associado a uma chance 2,5 vezes maior de tolerância à glicose diminuída [RC (IC 95\%): 2,53 $(1,34-4,79), p$ tendência $=0,005]$ em relação ao menor tercil de consumo ajustado pelas calorias totais e demais fatores de risco potenciais, sugerindo uma influência dos alimentos fonte de fibra no risco das alterações metabólicas (38).

Um estudo prospectivo conduzido em 37.000 adultos durante 4 anos de seguimento, o Melbourne Collaborative Cohort Study, observou um risco 40\% maior de incidência de diabetes no quartil superior de consumo de pão branco quando comparado ao quartil inferior independente de outros fatores de risco potenciais (39).

As relações entre o consumo de cereais refinados e risco de alteração da homeostase glicêmica (AHG) também foram observadas entre os homens do Health Professional Study e entre as mulheres do Nurses' Health Study, em que o maior consumo de pão branco, arroz polido, batata frita e cozida representou um incremento no risco de diabetes $(24,35)$.

\section{Frutose}

A frutose é um carboidrato naturalmente encontrado em frutas e vegetais ou artificialmente adicionado em bebidas e alimentos. Apesar da literatura sugerir que a substituição da sacarose por frutose possa promover menor resposta glicêmica e insulinêmica pós-prandial em indivíduos normais e portadores de diabetes com bom controle metabólico (40), evidências sugerem que o uso da frutose não deveria ser recomendado para indivíduos portadores de diabetes com controle metabólico precário, contribuindo para hiperglicemia (41).

A hipertrigliceridemia e glicosilação de proteínas provenientes do maior consumo de frutose vêm sendo sugeridas em alguns estudos. Entretanto, como este carboidrato é rapidamente convertido em glicose no fígado, este efeito seria observado na condição de um consumo elevado de frutose (41). Um ensaio clínico cruzado conduzido em 12 mulheres demonstrou que uma dieta isocalórica com elevados teores de frutose (30\% dos carboidratos adicionados em bebidas) resultou em redução estatisticamente significante na glicemia, insulinemia de jejum e leptina (associado ao aumento na concentração sérica de triglicérides) quando comparada a uma dieta com teor elevado de glicose ( $30 \%$ dos carboidratos adicionado em bebidas). $\mathrm{O}$ ba- lanço controlado pelo sistema nervoso central é influenciado pela insulina e leptina (42), assim a dieta rica em frutose representaria um fator de risco potencial para o ganho de peso corporal. $\mathrm{O}$ peso excessivo, por sua vez, está relacionado à maior síntese de ácidos graxos não esterificados, o que intensificaria a resistência à insulina devido à sua ação nos receptores periféricos.

Uma análise transversal dos dados do Nurses' Health Study demonstrou uma relação positiva entre o consumo de frutose com a concentração de peptídeo C plasmático, importante marcador da síntese da insulina e resistência periférica, aumentando o risco de diabetes (43). As principais fontes de frutose observadas foram suco de frutas, maçã, bebidas com cafeína e uva-passa. Verificou-se uma relação positiva entre o consumo de sucos de frutas e a concentração de peptídeo C plasmático, independente do valor calórico da dieta, consumo habitual de álcool, idade, tabagismo, IMC, prática de atividades físicas, HA e condição de menopausa, sugerindo efeitos adversos do consumo excessivo de sucos de frutas em relação ao risco para diabetes em mulheres.

Uma análise prospectiva das mulheres do Nurses' Health Study II verificou que o maior consumo (uma porção/dia) de bebidas adocicadas (sucos e refrigerantes, ricos em frutose e sacarose) representou um risco para diabetes duas vezes superior [RC 2,00 (IC 95\% 1,33; 3,01)] ao observado entre mulheres que relataram menor consumo destas bebidas (uma porção mensal), após 8 anos de seguimento (36).

\section{Sacarose}

A relação entre o consumo de açúcar da dieta e risco para o diabetes tem sido considerada controversa. Embora a Organização Mundial da Saúde recomende um consumo inferior a $10 \%$ das calorias totais para a prevenção de doenças crônicas (2), diversos estudos não observaram associação entre consumo de sacarose e resistência à insulina $(27,44,45)$ ou incidência de diabetes $(46)$. Uma redução de $30 \%$ na incidência do diabetes foi observada entre 37.000 adultos no quartil superior de consumo de açúcares (sacarose, alimentos com adição de sacarose e frutose) quando comparado ao menor aporte (mediana da população de $100 \mathrm{~g} / \mathrm{dia}$ ) após 4 anos de seguimento (36). Estes dados sugerem a possibilidade de sub-relato ou redução do consumo de açúcar por indivíduos portadores de fatores de risco para o diabetes.

\section{Lactose}

Evidências epidemiológicas da associação entre a lactose da dieta habitual e risco para diabetes são escassas. Um efeito protetor do maior consumo de leite para 
obesidade foi observado em um estudo conduzido entre 462 adultos iranianos. O quartil superior de consumo de leite ( $>3$ porções/dia) representou uma chance $80 \%$ menor no risco de excesso de peso IMC $\geq 25 \mathrm{~kg} / \mathrm{m}^{2}$ [RC $\left.0,78(0,43 ; 0,92)\right]-$ e obesidade - IMC $\geq 30 \mathrm{~kg} / \mathrm{m}^{2}[$ RC $0,73(0,40 ; 0,83)]-$ quando comparado ao menor quartil de consumo (< 1,5 porções/dia), independentemente da idade, atividade física, valor calórico total, carboidratos, fibras e proteínas da dieta habitual (47).

Um estudo prospectivo conduzido com 3.000 adultos observou um efeito protetor do maior consumo de laticínios para a incidência de síndrome metabólica entre adultos com sobrepeso, após 10 anos de seguimento. Neste estudo, cada porção de leite consumida ao dia representou uma redução de $21 \%$ no risco para a síndrome $[\mathrm{RC} 0,79(0,70 ; 0,88)]$, independentemente da idade, sexo, raça, tabagismo, consumo de álcool, atividade física, escolaridade, consumo calórico total, fibras e proteínas da dieta habitual (48).

No Estudo de Diabetes e Doenças Associadas em Nipo-Brasileiros de Bauru (28), observou-se uma relação inversa entre o consumo de laticínios com a glicemia de jejum $[\beta 1$ do $\log$ de glicemia (IC 95\%I): $-0,0001075(-0,000183 ;-0,000032)]$. Além disso, observou-se uma redução média de $40 \%$ no risco de diabetes para o consumo do maior tercil do grupo de laticínios (média de $200 \mathrm{~g} /$ dia) em relação ao primeiro tercil de consumo $[\mathrm{RC} 0,59(0,35 ; 1,00) ; \mathrm{p}$ tendência $=0,04]$, independentemente da idade, geração, sexo, tabagismo, prática de atividade física de lazer, obesidade abdominal, dislipidemia, hipertensão, escolaridade, consumo de fibras, colesterol e calorias totais da dieta habitual. Um relação positiva entre o consumo de laticínios e insulinemia também foi verificada $[\beta]$ do log de insulina (IC 95\%I): 0,0005533 (0,000018; $0,001089)$ ], concordando com dados prévios de estudos experimentais que demonstraram uma ação direta dos aminoácidos contidos na proteína do leite no incremento da síntese de insulina (9). Entre as mulheres do Nurses' Health Study e homens do Health Professional Study foi também observada uma relação protetora entre o consumo de iogurte para a incidência de diabetes $(24,35)$.

As evidências epidemiológicas da associação entre a lactose da dieta e diabetes são ainda inconsistentes. Embora os laticínios sejam as principais fontes alimentares de lactose da dieta habitual, estes são ricos em cálcio, potássio e magnésio - minerais possivelmente relacionados ao menor risco de outras doenças crônicas, tais como a hipertensão e o doença isquêmica do coração (49). As evidências epidemiológicas da relação entre lactose da dieta e risco para diabetes são inconsistentes. Em análise transversal dos dados provenientes do Inter99, estudo conduzido entre 5.675 dinamarqueses, uma relação positiva entre a lactose da dieta e HOMA [ $\beta 1$ (IC 95\%): 1,02 (1,01-1,02)] foi observada, independentemente do sexo, idade, tabagismo, atividade física, IMC, circunferência de cintura e consumo calórico total (27). Entretanto, este efeito pode ser conseqüente da maior síntese de insulina promovida pela lactose.

\section{DISCUSSÃO}

No panorama mundial de vertiginoso incremento na freqüência de doenças crônicas não-transmissíveis, como o diabetes, torna-se imprescindível o desenvolvimento de linhas de investigação da relação entre o estilo de vida e a gênese da doença. Embora o papel de uma dieta com baixo índice glicêmico no controle metabólico de indivíduos portadores de diabetes seja bem estabelecido, evidências sobre a associação entre a quantidade e qualidade dos carboidratos consumidos e prevenção primária do diabetes são controversas (4). No presente estudo de revisão, discutimos as evidências epidemiológicas da associação entre a qualidade dos carboidratos da dieta habitual e risco de diabetes e doenças associadas.

A carência de dados do índice glicêmico dos alimentos/preparações, ausência de instrumentos específicos para sua avaliação, diferentes fontes alimentares segundo localização geográfica, predisposição genética e resistência à insulina das populações avaliadas são possíveis hipóteses de explicação para resultados controversos para a relação entre qualidade dos carboidratos da dieta e risco para diabetes. A influência do peso corporal e sensibilidade à insulina na relação entre dieta habitual e risco para doenças crônicas pode ser fundamentada pelos resultados do Women's Health Study (13), cujo risco para DCV no maior consumo de índice glicêmico foi $50 \%$ superior entre mulheres portadoras de excesso de peso (IMC $>29 \mathrm{~kg} / \mathrm{m}^{2}$ ) quando comparado ao risco observado entre mulheres eutróficas (IMC entre 23 e $29 \mathrm{~kg} / \mathrm{m}^{2}$ ). No Brasil, dados do Estudo de Diabetes e Doenças Associadas em Nipo-Brasileiros sugerem relação positiva entre o IG da dieta e glicemia de jejum em mulheres com IMC superior a $23 \mathrm{~kg} / \mathrm{m}^{2}(28)$.

Uma limitação dos estudos em epidemiologia nutricional é a possível influência de outros fatores dietéticos e de estilo de vida na relação entre o consumo de nutrientes e ocorrência da doença, sugerindo 
a relevância de estudos que considerem os alimentos e padrões alimentares como um todo. Nos estudos conduzidos para avaliar a relação da qualidade dos carboidratos no risco de diabetes, verifica-se uma possível influência dos reduzidos teores de magnésio usualmente observados em dietas de elevado índice glicêmico na determinação da doença. Embora uma relação de risco entre o índice glicêmico da dieta habitual para o diabetes tenha sido observado em alguns estudos prospectivos, Nurses' Health Study I (24) e Health Professional Study (35), esta associação tornou-se estatisticamente significante somente após o ajuste pelos cereais integrais, principal fonte de magnésio da dieta americana. Por outro lado, no Iowa Women's Health Study (32) e Framingham Offspring Cohort (21), a associação entre o índice glicêmico e incidência do diabetes não foi independente do magnésio da dieta.

Os resultados de estudos epidemiológicos que investigaram a relação entre o consumo de fibras e risco de diabetes são igualmente controversos. Nenhuma relação foi observada entre o consumo de fibras provenientes de frutas e vegetais e incidência de DM em alguns estudos, embora um efeito protetor para as fibras de cereais tenha sido verificado (24,32-35). Entretanto, a associação entre o consumo de fibras de cereais e ocorrência de diabetes em alguns estudos não foi independente do magnésio da dieta $(21,24,31)$, sugerindo que o efeito protetor seja atribuído ao magnésio contido nestes alimentos. $\mathrm{O}$ efeito protetor do magnésio para o diabetes vem sendo apontado por estudos epidemiológicos, cujas hipóteses de explicação estariam relacionadas tanto à sua função como co-fator de enzimas envolvidas no metabolismo dos carboidratos como a um efeito direto na síntese e ação periférica da insulina $(32,50-52)$.

Estudos epidemiológicos que investigaram a relação entre o consumo de laticínios e ocorrência de doenças crônicas sugerem um efeito protetor para obesidade e síndrome metabólica, independente de fatores de risco potenciais $(47,48)$. No Estudo de Diabetes e Doenças Associadas em Nipo-Brasileiros observou-se proteção do maior consumo de laticínios para o diabetes em relação ao tercil inferior de consumo (28). Além disso, observou-se uma relação inversa entre o consumo de leite/derivados e glicemia de jejum, assim como a relação positiva com a insulinemia, fundamentando a hipótese de estímulo direto dos aminoácidos contidos na proteína do leite na síntese de insulina, previamente sugeridos por estudos experimentais (9). Entretanto, leites, queijos e iogurtes são fontes importantes de cálcio, potássio e magnésio da dieta e o efeito protetor para os distúrbios do metabolismo da glicose pode estar relacionado ao seu maior aporte. As evidências disponíveis do papel da lactose da dieta na determinação do diabetes são insuficientes, sendo necessário o desenvolvimento de estudos prospectivos que avaliem o efeito do consumo de laticínios, lactose, cálcio, magnésio e potássio na ocorrência da doença.

O consumo de sacarose vem sendo desencorajado para a prevenção primária de doenças crônicas, tais como obesidade e diabetes. Embora alguns estudos apontem a ausência de relação de risco de seu consumo para a resistência à insulina $(27,44,45)$ e diabetes $(28,46)$, sua associação com a obesidade vem sendo fundamentada por diversos estudos epidemiológicos (2). Na cultura popular, a redução do consumo de açúcares e sua substituição por adoçantes dietéticos são consideradas medidas eficazes tanto na prevenção como no controle do excesso de peso e diabetes (53), sugerindo a possibilidade de efeito de causalidade reversa ou restrição do consumo de açúcar entre indivíduos portadores de fatores de risco para o diabetes. Segundo Mann (54), em recente artigo de revisão, dados provenientes de estudos ecológicos que demonstraram uma associação positiva entre o consumo de sacarose e ganho de peso corporal, ensaios clínicos aleatorizados que verificaram impacto na perda de peso pela substituição de carboidratos simples por complexos, estudos transversais ou prospectivos que observaram um risco de ganho de peso para o maior consumo de açúcares ou bebidas adocicadas são evidências suficientes da necessidade da adoção de medidas preventivas de saúde pública na contenção do consumo de açúcar.

A associação entre o consumo de frutose e risco de doenças crônicas tem sido pouco explorada em estudos epidemiológicos. Uma relação positiva entre a frutose da dieta e níveis séricos de leptina e insulina foi observada em estudo prévio, sugerindo um possível efeito no ganho de peso corporal (42). Além disso, uma relação positiva com a concentração de peptídeo C plasmático, marcador de risco para o diabetes, foi verificada em recente análise de um grande estudo americano (43). Estes resultados sugerem um possível efeito da frutose da dieta na etiologia de doenças crônicas, sendo necessário um maior número de estudos na elucidação desta associação.

A alteração da qualidade dos carboidratos da dieta habitual observada em diversos países vem sendo apontada como um dos fatores determinantes do incremento da freqüência do diabetes. Uma análise ecológica da associação entre a qualidade dos carboidratos consumidos na dieta americana na prevalência da doença observada nos Estados Unidos nos últi- 
mos 20 anos, verificou que a tendência do incremento no consumo de carboidratos refinados [provenientes do melado de milho (syrup, em inglês)], altamente concentrado em frutose e sacarose, em detrimento do consumo de alimentos naturalmente ricos em fibras estava fortemente relacionado à maior ocorrência do diabetes tipo 2 (55). No Brasil, o envelhecimento populacional associado ao aumento da freqüência do excesso de peso, estilo de vida sedentário e modificações no padrão alimentar, como o aumento do consumo de açúcares e refrigerantes em detrimento das frutas, verduras e legumes, têm sido apontados como possíveis fatores envolvidos no incremento da ocorrência do diabetes nos últimos anos (56).

Evidências provenientes de ensaios clínicos aleatorizados com intervenção nutricional para prevenção primária do diabetes conduzidos entre indivíduos portadores de fatores de risco fundamentam a hipótese da eficácia da intervenção no estilo de vida com ênfase na prática de atividades físicas e uma alimentação rica em cereais integrais, frutas, verduras e legumes em detrimento do consumo de cereais refinados - na redução do risco para o diabetes, sendo estas medidas significativamente mais eficazes que a intervenção medicamentosa (57).

No Brasil, um ensaio clínico aleatorizado conduzido em adultos com sobrepeso em unidade básica de saúde verificou que 3 consultas individualizadas com o nutricionista - constando do incentivo ao consumo de cereais integrais, frutas, verduras, legumes, azeite de oliva e peixes em detrimento das carnes e cereais refinados - resultou em uma redução de $3,5 \%$ do peso corporal, 15\% do LDL-colesterol e manutenção dos níveis de HDL-colesterol no grupo intervenção após 6 e 12 meses de seguimento, sugerindo que a alteração da qualidade dos carboidratos e lipídeos da dieta associada à prática de atividades físicas pode repercutir em um importante impacto no controle de fatores de risco potenciais para o diabetes tipo $2(58,59)$.

\section{CONCLUSÃO}

As evidências epidemiológicas do papel da qualidade dos carboidratos na gênese e prevenção do diabetes ainda são inconsistentes. Embora resultados de alguns estudos indiquem uma possível associação entre dieta com elevados teores de índice glicêmico e pobre em fibras de cereais e maior risco para diabetes, há indícios de que esta relação seja mediada pelos baixos teores de magnésio deste padrão de consumo alimentar, sugerindo a relevância de se considerar os alimentos e padrões alimentares como um todo em investigações sobre fatores determinantes de doenças crônicas.

Os estudos discutidos na presente revisão sugerem que uma dieta rica em cereais integrais e vegetais, fontes naturais de magnésio e fibras, e pobre em cereais refinados e sacarose, possa exercer um papel protetor para o diabetes. Um efeito benéfico na gênese de distúrbios do metabolismo da glicose parece estar relacionado ao maior consumo de laticínios. Em relação à frutose, as evidências ainda são escassas mas sugerem uma relação de risco para o diabetes $\mathrm{e}$ doenças associadas, indicando a necessidade de recomendações do consumo moderado de alimentos com elevadas concentrações de frutose, tais como frutas passas e sucos de frutas.

Fontes alimentares distintas de fibras e índice glicêmico consumidas por indivíduos com diversificada susceptibilidade genética, peso corporal e resistência à insulina podem influenciar a resposta metabólica individual, fato que pode explicar em parte os controversos resultados dos estudos epidemiológicos disponíveis, enfatizando a necessidade de maior número de estudos prospectivos para a elucidação das relações entre o consumo alimentar e risco para diabetes, ferramenta fundamental no planejamento de medidas de prevenção.

Evidências provenientes de ensaios clínicos aleatorizados para a prevenção primária do diabetes 2 sugerem que orientações nutricionais enfocando a qualidade dos carboidratos e lipídeos da dieta, como o estímulo ao consumo de cereais integrais, frutas, verduras, legumes, azeite de oliva e peixes, em detrimento do consumo de carnes e cereais refinados associadas ao incentivo da prática de atividades físicas podem produzir um importante impacto na prevenção do diabetes tipo 2 em indivíduos portadores de fatores de risco.

\section{REFERÊNCIAS}

1. King $\mathrm{H}$, Aubert RE, Herman WH. Global Burden of Diabetes, 1995 - 2025. Diabetes Care 1998;21:1414-31.

2. World Health Organization. Food and Agriculture Organization. Joint WHO/FAO expert consultation. Diet, nutrition and the prevention of chronic diseases. Geneva: WHO/FAO; 2003.

3. Venn BJ, Mann Jl. Cereal grains, legumes and diabetes. Eur J Clin Nutr 2004;58:1443-61.

4. Sheard NF, Clark NG, Brand-Miller JC, Franz MJ, Pi-Sunyer FX, Mayer-Davis E, et al. Dietary carbohydrate (amount and type) in the prevention and management of diabetes: A statement by the American Diabetes Association. Diabetes Care 2004;27:2266-71. 
5. Jenkins DJ, Wolever TM, Taylor RH, Barker HM, Fielden H, Baldwin JM, et al. Glycaemic index of foods: a physiological basis for carbohydrate exchange. Am J Clin Nutr 1981;34:362-6.

6. Jenkins DJA, Kendall CWC, Augustin LSA, Franceschi S, Hamidi M, Marchie A, et al. Glycemic index: overview of implications in health and disease. Am J Clin Nutr 2002;76:266-73.

7. Foster-Powell K, Holt SHA, Brand-Miller JC. International table of glycemic index and glycemic load values: 2002. Am J Clin Nutr 2002;76:55-6.

8. Collier $G$, O'Dea K. The effect of co-ingestion of fat on the glucose, insulin and gastric inhibitory polypeptide responses to carbohydrate and protein. Am J Clin Nutr 1983:37:941-4.

9. Wolever TMS, Jenkins DJA, Jenkins AL, Jesse RG. The glycemic index: methodology and clinical implications. Am J Clin Nutr 1991:54:846-54.

10. Menezes EW, Lajolo FM. Utilização do amido de leguminosas. Arch Latinoam Nutr 1995;45(suppl. 1):270-2.

11. Würsch P, PI-Sunyer EX. The role of viscous soluble fiber in the metabolic control of diabetes. Diabetes Care 1997:20:1774-89

12. Storlien LH, Kraegen EW, Jenkins AB, Chisholm DJ. Effect of sucrose vs. satarch diets on in vivo insulin action, thermogenesis and obesity in rats. Am J Clin Nutr $1988: 47: 420-7$

13. Liu S, Willett WC, Stampfer MJ, Hu FB, Franz M, Sampson $L$, et al. A prospective study of dietary glycemic load carbohydrate intake, and risk of coronary heart disease in US women. Am J Clin Nutr 2000;71:1455-61

14. Willett WC, Manson J, Liu S. Glycemic index, glycemic load, and risk of type 2 diabetes. Am J Clin Nutr 2002;76:274-80.

15. Ludwig DS. Dietary Glycemic index and obesity. J Nutr 2000; 130 (suppl. 2):280-3.

16. Brand-Miller JC, Holt SHA, Pawlak DB, McMillan J. Glycemic index and obesity. Am J Clin Nutr 2002;76(suppl. 1):281-5.

17. Leeds AR. Glycemic index and heart disease. Am J Clin Nutr 2002;76(suppl. 1):286-9.

18. Liu S, Manson JE, Stamfer MJ, Holmes MD, Hu FB, Hankinson $\mathrm{SE}$, et al. Dietary glycemic load assessed by foodfrequency questionnaire in relation to plasma high-density-lipoprotein cholesterol and fasting plasma triacylglycerol in postmenopausal women. Am J Clin Nutr 2001;73:560-6.

19. Amano Y, Kawakubo K, Lee JS, Tang AC, Sugiyama M, Mori K. Correlation between dietary glycemic index and cardiovascular disease risk factors among Japanese women. Eur J Clin Nutr 2004:58:1472-8.

20. Liu S, Manson JE, Buring JE, Stampfer MJ, Willett WC, Ridker PM. Relation between a diet with a high glycemic load and plasma concentrations of high-sensitivity Creactive protein in middle-aged women. Am J Clin Nutr 2002; 75:492-8

21. Mckeown NM, Meigs JB, Liu S, Saltzman E, Wilson PWF, Jacques PF. Carbohydrate nutrition, insulin resistance, and the Prevalence of the Metabolic Syndrome in the
Framingham Offspring Cohort. Diabetes Care 2004:27:538-46.

22. Parks EJ, Hellerstein MK. Carbohydrate-induced hypertriacylglycerolemia: historical perspective and review of biological mechanisms. Am J Clin Nutr 2000;71:412-33.

23. Brand-Miller JC, Hayne S, Petocz P, Colagiuri S. Lowglycemic index diets in the management of diabetes. Diabetes Care 2003;26:2261-7.

24. Salmeron J, Manson JE, Stampfer MJ, Colditz GA, Wing AL, Willett WC. Dietary fiber, glycemic load, and risk of non-insulin-dependent diabetes mellitus in women. JAMA 1997:277:472-7.

25. Wolever TMS, Mehling C. Long-term effect of varying the source or amount of dietary carbohydrate on postprandial plasma glucose, insulin, triacylglycerol, and free fatty acid concentrations in subjects with impaired glucose tolerance. Am J Clin Nutr 2003:77:612-21.

26. Jenkins DJ, Wolever TM, Ocana AM, Vuksan V, Cunnane $S C$, Jenkins $M$, et al. Metabolic effects of reducing rate of glucose ingestion by single bolus versus continuous sipping. Diabetes 1990;39:775-81.

27. Lau C, Ferch K, Glümer C, Tetens I, Pedersen O, Carstensen B, et al. Dietary glycemic index, glycemic load, fiber, simple sugars, and insulin resistance. Diabetes Care 2005;28(6): 1397-403.

28. Sartorelli DS. Índice glicêmico da dieta habitual e alteração da homeostase glicêmica em nipo-brasileiros de Bauru. São Paulo; 2005. (Tese de Doutorado - Universidade de São Paulo). <http:// www.teses.usp.br>.

29. Mckeown NM, Meigs JB, Liu S, Wilson PWF, Jacques PF. Whole-grain intake is favorably associated with metabolic risk factors for type 2 diabetes and cardiovascular disease in the Framingham Offspring Study. Am J Clin Nutr 2002;76:390-8.

30. Jensen MK, Baneriee PK, Hu FB, Franz M, Sampson L Gronbek M, et al. Intakes of hole grains, bran, and germ and the risk of coronary heart disease in men. Am J Clin Nutr 2004;80:1492-9.

31. Fung $T$, Hu FB, Pereira MA, Liu S, Stampfer MJ, Colditz $G A$, et al. Whole-grain intake and the risk of type-2 diabetes: a prospective study in men. Am J Clin Nutr 2002; 76:535-40.

32. Meyer KA, Kushi LH, Jacobs DR, Slavin J, Sellers TA, Folsom AR. Carbohydrates, dietary fiber, and incident type 2 diabetes in older women. Am J Clin Nutr 2000;71:921-30.

33. Stevens J, Ahn K, Juhaeri, Houston D, Steffan L, Couper D. Dietary fiber intake and glycemic index and incidence of diabetes in African-American and white adults. Diabetes Care 2002;25:1715-21.

34. Montonen J, Knekt $P$, Järvinen R, Aromaa A, Reunanen A. Whole-grain and fiber intake and the incidence of type 2 diabetes. Am J Clin Nutr 2003;77:622-9.

35. Salmeron J, Ascherio A, Rimm EB, Colditz GA, Spiegelman D, Jenkins DJ, et al. Dietary fiber, glycemic load, and risk of NIDDM in men. Diabetes Care 1997;20:545-50.

36. Schulze MB, Manson JE, Ludwig DS, Colditz GA, Stampfer MJ, Willett WC, et al. Sugar-sweetened beverages, weight gain, and incidence of type 2 diabetes in young and middle-aged women. JAMA 2004: 292:927-34. 
37. Liese AD, Roach AK, Sparks KC, Marquart L, D'Agostino RB, Mayer-Davis EJ. Whole-grain intake and insulin sensitivity: the Insulin resistance Atherosclerosis Study. Am J Clin Nutr 2003:78:965-71.

38. Sartorelli DS, Freire RD, Ferreira SR, Cardoso MA; for the Japanese-Brazilian Diabetes Study Group. Dietary fiber and glucose tolerance status in Japanese-Brazilians. Diabetes Care 2005:28:2240-2.

39. Hodge AM, English DR, O’Dea K, Giles GG. Glycemic index and dietary fiber and the risk of type 2 diabetes. Diabetes Care 2004:27:2701-6.

40. Heacock PM, Hertzler SR, Wolf BW. Fructose prefeeding reduces the glycemic responses to a high-glycemic index, starchy food in humans. J Nutr 2002;132:2601-4.

41. Uusitupa MIJ. Fructose in the diabetic diet. Am J Clin Nutr 1994;59(suppl. 3):753-7.

42. Teff KL, Elliott SS, Tschöp M, Kieffer TJ, Rader D, Heiman $M$, et al. Dietary fructose reduces circulating insulin and leptin, attenuates postprandial suppression of ghrelin, and increases triglycerides in women. J Clin Endocrino Metab 2004;89:2963-72.

43. Wu T, Giovannucci E, Pischom T, Hankinson SE, Ma J, Rifai $\mathrm{N}$, et al. Fructose, glycemic load and quantity and quality of carbohydrate in relation to plasma C-peptide concentrations in US women. Am J Clin Nutr 2004:80:1043-9.

44. Daly M. Sugars, insulin sensitivity, and postprandial state. Am J Clin Nutr 2003;78(suppl. 4):865S-72S

45. Sevak L, McKeigue PM, Marmot MG. Relationship of hyperinsulinemia to dietary intake in south Asian and European men. Am J Clin Nutr 1994:59:1069-74.

46. Janket SJ, Manson JE, Sesso H, Buring JE, Liu S. A prospective study of sugar intake and risk of type 2 diabetes in women. Diabetes Care 2003:26:1008-15.

47. Mirmiran P, Esmaillzadeh A, Azizi F. Dairy consumption and body mass index: an inverse relationship. Int J Obes Relat Metab Disord 2005;29:1 15-21

48. Pereira MA, Jacobs DR, Horn LV, Slattery ML, Kartashov Al, Ludwig DS. Dairy consumption, obesity, and the insulin resistance syndrome in young adults: The CARDIA Study. JAMA 2002;287:2081-9.

49. Aschiero A, Rimm EB, Hernán MA, Giovannucci EL, Kawachi I, Stampfer MJ, et al. Intake of potassium, magnesium, calcium, and fiber and risk of stroke among US men. Circulation 1998:98:1198-204.
50. Colditz AC, Manson J, Stampfer MJ, Rosner B, Willett WC, Speizer FE. Diet and risk of clinical diabetes in women. Am J Clin Nutr 1992;55:1018-23.

51. Kao WHL, Folsom AR, Nieto FJ, Mo JP, Watson RL, Brancati FL. Serum and dietary magnesium and the risk for type 2 diabetes mellitus. Arch Intern Med 1999;159:2151-9.

52. Lopez-Ridaura R, Willett WC, Rimm EB, Liu S, Stampfer $\mathrm{MJ}$, Manson JE, et al. Magnesium intake and risk of type 2 diabetes in men and women. Diabetes Care 2004; $27: 134-40$.

53. Castro AGP, Franco JL. Caracterização do consumo de adoçantes alternativos e produtos dietéticos por indivíduos diabéticos. Arq Bras Endocrinol Metab 2002:46:280-7

54 Mann J. Free sugars and human health: sufficient evidence for action? Lancet 2004;363:1068-9.

55. Gross LS, Li L, Ford ES, Liu S. Increased consumption of refined carbohydrates and epidemic of type 2 diabetes in the United states: an ecologic assessment. Am J Clin Nutr 2004;79:774-9.

56. artorelli DS, Franco LJ. Tendências do diabetes mellitus no Brasil: o papel da transição nutricional. Cad Saúde Pública 2003; 19(Supl. 1):S29-S36.

57. Sartorelli DS, Franco LJ, Cardoso MA. Intervencão nutricional e prevenção primária do diabetes mellitus tipo 2: uma revisão sistemática. Cad Saúde Pública 2006:22:718

58. Sartorelli DS, Sciarra EC, Franco LJ, Cardoso MA. Primary prevention of type 2 diabetes through nutritional counseling. Diabetes Care 2004;27:3019.

59. Sartorelli DS, Sciarra EC, Franco LJ, Cardoso MA. Beneficial effects of short-term nutritional counseling at the primary health care level among Brazilian overweight adults. Public Health Nutr 2005;8:820-5

\section{Endereço para correspondência:}

Daniela Saes Sartorelli

Departamento de Medicina Social

Faculdade de Medicina de Ribeirão Preto

Universidade de São Paulo (USP)

Av. Bandeirantes 3900

14049-900 Ribeirão Preto, SP

E-mail: sartorellidaniela@hotmail.com 